\title{
An experimental study of liquid unloading in the curve section of horizontal gas wells
}

\author{
Shuzhe Shi ${ }^{1, *}$, Guoqing $\mathrm{Han}^{2}$, Bohong $\mathrm{Wu}^{1}$, Kangtai $\mathrm{Xu}^{3,{ }^{*}}$, Zhun $\mathrm{Li}^{4}$, and $\mathrm{Ke} \mathrm{Sun}^{2}$ \\ ${ }^{1}$ The Research Institute of Petroleum Exploration and Development, 100083 Beijing, PR China \\ ${ }^{2}$ College of Petroleum Engineering, China University of Petroleum, 102249 Beijing, PR China \\ ${ }^{3}$ Hebei Petroleum University of Technology, 071000 Hebei, PR China \\ ${ }^{4}$ CNOOC Research Institute, 100010 Beijing, PR China
}

Received: 17 March 2021 / Accepted: 9 September 2021

\begin{abstract}
Liquid unloading is a very common and important issue in horizontal gas wells, and the presence of curve sections increases the complexity of the phenomenon and its study. Liquid loading in a gas well will sharply reduce production, therefore, the liquid-unloading onset of different curved pipes is essential to gas production. In this work, liquid-unloading onset experiments were conducted in curved pipes with different curvatures. Then, the critical gas velocity $V_{\mathrm{sgCR}}$ can be determined according to the measured pressure gradients, liquid holdup, and liquid film reversal. This work analyzes the factors which will lead to the liquid unloading and explores the trend of the pipe curvature's influence on the liquid unloading under laboratory conditions. The experimental results show that the critical gas velocity rises with the increase of pipe curvature, the increase is mainly due to the centrifugal force. The present work also compares the predicted results of the OLGA model and Beggs-Brill model with experimental data. The comparison results indicate that both models fit relatively well to the experimental data at the low superficial gas velocity, and both models have poor performance at high superficial gas velocity. The OLGA model fits the experimental data better than the Beggs-Brill model at high superficial gas velocity. The error analysis shows that most of the predicted data is not in good agreement with experimental data. Some errors between experimental data and calculation results are out of the range of $50 \%$.
\end{abstract}

\section{Nomenclature}

$p \quad$ Pressure

$z \quad$ Pipe axial direction

$V_{\mathrm{sgCR}} \quad$ Critical gas velocity

$F_{g} \quad$ Gas drag force

g Gravitational acceleration

$F_{c} \quad$ Centrifugal force

$r \quad$ The curvature radius of pipe

$V_{f} \quad$ Liquid film velocity

$R \quad$ Pipe radius

$f_{l} \quad$ Interfacial friction factor

\section{Greek letters}

$\begin{array}{ll}\rho & \text { Density } \\ \theta & \text { Angle between the pipe and the horizon } \\ \delta & \text { Liquid film thickness }\end{array}$

\footnotetext{
* Corresponding authors: shishuzhe@petrochina.com.cn, 303070516@qq. com; xukangtai123@126.com
}

\author{
Subscripts \\ $l \quad$ Liquid phase \\ $g \quad$ Gas phase
}

\section{Introduction}

Liquid loading/unloading in gas wells is always an important issue in the oil/gas industry, which may prevent gas production and even shut down the gas well, which will cause economic losses. During the gas production process, the liquid phase exists in the well as liquid film and droplets which move along the tube, the gas phase exists in the well as gas core which carries the liquid droplets at the center of the tube. The gas velocity is much higher than the liquid velocity (Lea et al., 2008). The appearance of liquid loading in a gas well means the gas velocity is insufficient to carry liquid to the wellhead and the production of this well will reduce and even stop entirely.

The key issue of liquid unloading is the critical gas velocity (Zhou et al., 2016; Landjobo Pagou et al., 2020) along 
with the tubing. The reservoir pressure will decline as the oil/gas production continues, which will lead to the low gas rate and will cause the backflow of liquid and cause liquid accumulation in the gas well (Lea and Nickens, 2004; Zhou et al., 2019). There are primarily three methods to determine the critical gas velocity, which are the timing of liquid film reverse, pressure gradient change and liquid holdup change in the gas well.

The previous studies mainly focus on vertical wells and inclined wells (Klumpner and Blaabjerg, 2002; Guner et al., 2015; Pagan and Waltrich, 2016; Shekhar et al., 2017). In recent years, the exploitation of shale gas reservoirs and coal-bed gas reservoirs has got a lot of attention in China. Accordingly, the multi-fractured horizontal well is often applied in the exploitation process. Unlike vertical and inclined wells, the horizontal well has plenty of curve sections. The curve sections have different scales, that is to say, different pipe curvatures. The flow in the curve section is more complicated than it is in a straight section. Therefore, the liquid unloading in a curve section must be studied.

\subsection{Liquid loading/unloading models}

Turner et al. (1969) were the earliest researchers who proposed a model to predict the onset of liquid loading. Their model is based on the mechanical equilibrium between the liquid phase and the gas phase. Their main correlation is the semi-empirical equation, which is based on the critical Weber number and the drag coefficient. Their velocity equation was obtained by using the liquid droplet breakup mechanics. This equation needs an adjustment of $20 \%$ to ensure all the liquid drops are removal. Their model can not only be used in a common geometry pipe but also in the annulus pipe and others.

Coleman et al. (1991) proposed the critical gas velocity from the Turner et al. (1969) model without the adjustment and compared it with field results. They found that the liquid-droplet model could not be used when there was slug flow in the well. Nosseir et al. (2000) came up with a new method to predict the critical gas velocity in a vertical pipeline. Their model eliminated the empirical adjustment which was originated from the Turner et al. (1969) model. Instead, they considered different flow patterns and flow conditions to convert the empirical equations to analytical equations.

Min et al. (2001) assumed that the liquid drops carried by the gas core tend to be flat-shaped based on the Turner et al. (1969) model. The results of the proposed new equations for continuous liquid removal from gas wells are smaller than that of conventional Turner's equation, and they analyzed different factors that affect liquid removal. van't Westende et al. (2007) conducted some liquid-loading experiments of dispersed phase with the annular flow pattern in a vertical tube of $5 \mathrm{~cm}$ ID (Inner Diameter). They used PDA (Phase Doppler Anemometry) to measure the size and velocity of liquid drops entrained in the gas core. They found out that the dispersed phase would delay the onset of liquid loading, and the existence of liquid droplets would decrease the pressure gradient and the shear stress.
Wang et al. (2015) proposed a new model from Azzopardi et al.'s (1991) study with a new parameter $C_{k \text {,Wecrit }}$. In their study, $C_{k, \text { Wecrit }}$ and critical gas velocity $V_{\mathrm{sg}}$ increased with the pressure and the liquid volume. They applied plenty of gas well data to their model to verify it. Further, Riza et al. (2016) suggested a new model that coupled the reservoir and wellbore to simulate the liquid loading situation. They found the flow pattern transition from annular flow to slug flow leads to the downward film reversal, causing the liquid-loading in the well. Their model concluded that the most dominant parameters of the initiation of liquid-loading are the tubing diameter and the production index. Recently, Rodrigues et al. (2018) came up with a model to calculate the thickness of the liquid film at the well wall. The main point of their study was to add an equation that corrects the friction factor to the calculation process of the liquid film. Their model suggested that the liquid film has an increased roughness. They compared the model results to some previous experimental data, which indicated this model has an improvement in the pressure gradient calculation. But the liquid holdup calculated by their new model does not have a big difference.

Nodal analysis can also be used as an analyzing method in a liquid-loading gas well. Greene (1983) analyzed gas well performances through nodal analysis. He predicts the influence of declining reservoir pressure and changing tubing sizes on the gas well unloading.

Lea and Rowlan (2019) applied the nodal analysis method on the liquid-loading well and simulated different solutions of the liquid-loading situation by analyzing the tubing performance curve. They proposed the solutions by using smaller tubing, compression, surface choke, gas lift, plunger, foam, and pumping system to solve the liquid-loading problem. The application range of each method also has been discussed in their study.

\subsection{Liquid loading/unloading experiments}

Anderson and Russell (1970) used probes to detect the thickness of the liquid film, and they also studied the liquid drops' movement and the influence on the liquid film in a small diameter pipe. Williams et al. (1996) studied the liquid entrainment and droplet distributions in a horizontal tube with a diameter of $0.0953 \mathrm{~m}$. Results indicate that the liquid film distribution becomes more asymmetric as the increase of pipe diameter, and entrainment's change is not considerable.

Sawai et al. (2004) studied the pressure oscillatory characteristics between the different flow patterns. They conducted experiments in a $25.8 \mathrm{~mm}$ ID vertical pipe, and the cross-sectional liquid holdup and pressure gradient were measured at different locations. A simple model in this study is built by the friction multipliers and the liquid holdup equation, which can predict the frictional pressure gradient in these flow patterns. The experimental results showed that the increase of liquid wave velocity is much more than the normal liquid film velocity as the liquid flow rate decreased, which denoted that the frictional pressure gradient was predominantly influenced by the wave transition. Belfroid et al. (2008) made an advance in the 
inclination angle research. They used a $50 \mathrm{~mm}$ ID pipe to study the initiation of liquid loading with four different inclination angles. They found out that the main influence factor of the liquid loading initiation is liquid film transport, and they concluded two parameters that influenced the initiation of liquid loading. The first parameter is reservoir permeability. Liquid loading will happen much faster and more unstable in a high permeability reservoir. The second parameter is well deviation. As the rise of deviation angle, the film thickness at the bottom of the pipe increase which will influence the initiation of liquid loading. The maximum critical gas velocity will occur at the inclination of $50^{\circ}$, which is $40 \%$ higher than in a vertical pipe. The authors also used the inclination angle parameters to modify Turner's criterion of the liquid loading initiation.

Waltrich et al. (2015) conducted experiments to study the initiation of liquid-loading in a 2-in ID and $42 \mathrm{~m}$ long vertical pipe. They recorded pressure, temperature, and liquid holdup to analyze the liquid film reversal conditions and compared with previous models. The results suggested that one should not only consider Turner's or Wallis's criteria but also take into account the reservoir behavior when analyzing the liquid loading initiation. Different from Waltrich's research, Skopich et al. (2015) did some researches on the influence of pipe diameter on the initiation of liquid loading. 2-in and 4-in ID pipes were used in the two-phase flow experiments. The experimental results showed that the 2-in ID pipe has the largest critical gas velocity, and it decreases as the pipe ID increases. They found that the initiation of liquid film reversal is inconsistent with previous studies in the 2-in ID pipe case. The main reason for this situation is the incorrect flow-pattern prediction.

Therefore, the main object of this work is to investigate the onset of liquid unloading of different curved pipes. The pressure gradient and liquid holdup were measured, and water film reversal was recorded to determine the liquid unloading onset. The influences of the different pipe curvatures, superficial gas velocities, and superficial liquid velocities on the liquid unloading onset in the curved pipe were studied in this work. Then, the predicted results of the OLGA model and Beggs-Brill model were compared with experimental data to verify the models' accuracy of the curved pipe cases.

\section{The liquid unloading experiments}

\subsection{The experiments facility}

The experimental facility consists of several $1 \mathrm{~m}$ transparent acrylic resin horizontal pipes and three curved pipes (the curvature radius is $1 \mathrm{~m}, 1.5 \mathrm{~m}$, and $2 \mathrm{~m}$, respectively) with an inner diameter of $50 \mathrm{~mm}$, and the flow loop has a total of $10 \mathrm{~m}$ long, some of the experiment configurations are used in the previous work by the same authors (Shi et al., 2019). The schematic of the experiment facility is shown in Figure 1. The curved pipe and straight pipe are fixed on a steel holder to support the test pipeline, these pipes are connected with a flange, making it easy to change the curve section. All pipes in the test section have the same inner diameter and a straight pipe is installed before the curve section to reduce the inlet phenomena.

\subsection{The experiments arrangement}

In the experiment process, gas is injected by the air compressor and stored in a gas tank. The gas velocity is controlled by a magnetic valve as the gas goes out from the gas holder, then measured by a gas mass flowmeter in the range of $0.1-100 \mathrm{~m}^{3} / \mathrm{h}$ with an accuracy of $\pm 1 \%$ of the measurement range. The liquid is injected by a centrifugal pump from a water tank and measured by a flowmeter in the range of $0-1 \mathrm{~m}^{3} / \mathrm{h}$ with an accuracy of $\pm 1 \%$ of the measurement range. The gas and liquid streams converge in a mixer, and the air-water will be fully blended in the mixer and flow through the test section. The mixer is a tee-junction with the gas inlet extending into the main pipe. Besides, the extended gas pipe has some pores to make gas and liquid better mixing. Then the pressure of the curved pipe is measured by RoseMount pressure sensors in the range of $0-500 \mathrm{kPa}$, which are installed at the inlet, middle, and outlet of the curve pipe. As shown in Figure 2, the liquid holdup is measured by a conductivity proposed by Fossa (1998) and updated by Zhong et al. (2018), which is installed at the outlet of the curve pipe. This method is relatively simple and can record the real-time liquid holdup without breaking the flow pattern. The calibration of the conductivity is shown in Appendix. It is installed at the outlet of the curved pipe and the flow patterns and water film are recorded by a high-speed camera. The device uses a high-speed acquisition card to collect all the pressure and liquid holdup data, and displays and stores them in the computer. The water is then recycled to the water tank connected to the experimental test section outlet while the water tank is open to the atmosphere to vent the gas.

Before the formal experiments, repetitive tests have been made with three different liquid and gas velocities to verify the repeatability.

\section{Experimental results and analysis}

The experimental matrix is designed to include the slug flow pattern and annular flow pattern because the process of liquid unloading can be fully recorded. The superficial liquid velocities were set as $0.008 \mathrm{~m} / \mathrm{s}, 0.016 \mathrm{~m} / \mathrm{s}$ and $0.024 \mathrm{~m} / \mathrm{s}$ and the superficial gas velocities varied from $3.5 \mathrm{~m} / \mathrm{s}$ to $33.0 \mathrm{~m} / \mathrm{s}$. Curved pipes with $1 \mathrm{~m}, 1.5 \mathrm{~m}$ and $2 \mathrm{~m}$ curvature were tested.

\subsection{Test results for the curved pipe with $1 \mathrm{~m}$ curvature}

Based on the study by Sarica et al. (2013), the $V_{\mathrm{sgCR}}$ reaches the maximum at the inclined angle of $30^{\circ}$, therefore, a high-speed camera was used to record the liquid film flow reversal and two-phase flow patterns at the first part of the curve pipe. As observed in the $1 \mathrm{~m}$ curvature experiments, the liquid film moves downward at low superficial gas velocities $\left(V_{\mathrm{sg}}<17.2 \mathrm{~m} / \mathrm{s}\right)$. As the superficial gas velocity rises, the liquid film gradually stops falling back and even moves upward. For deciding the liquid-unloading initiations, liquid 


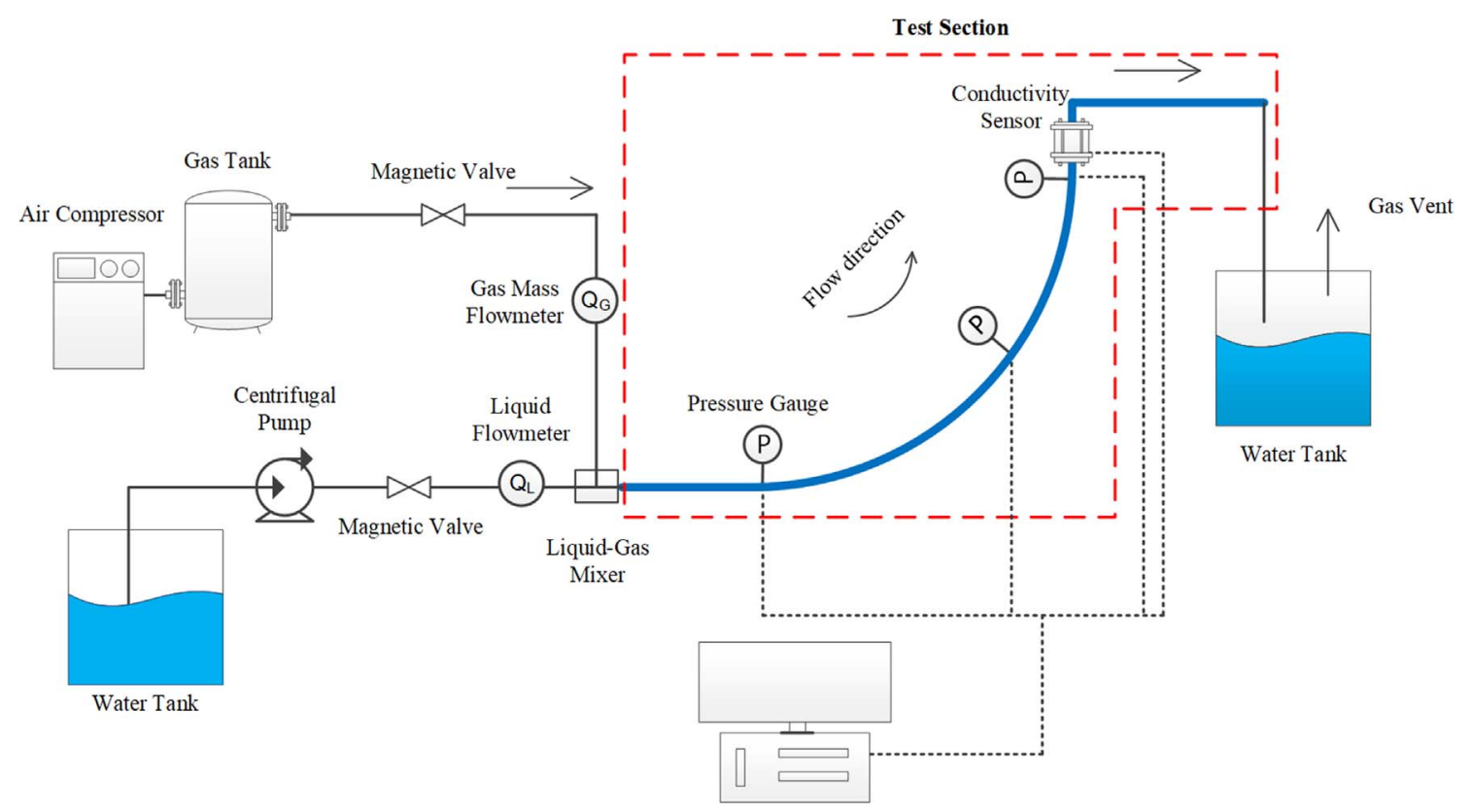

Fig. 1. Schematic illustration of the curved pipe experimental flow loop.
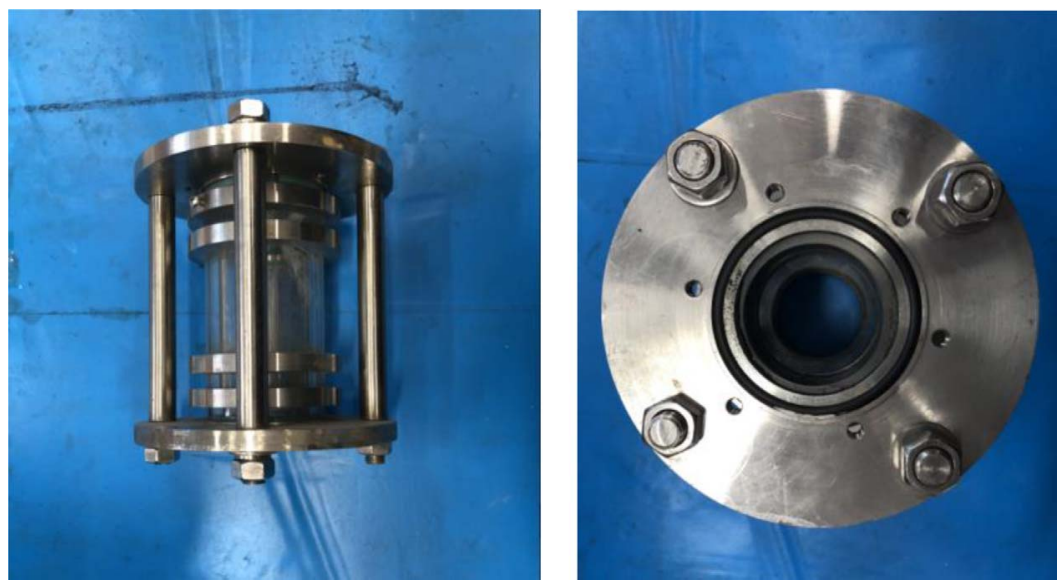

Fig. 2. The liquid holdup measurement unit.

film reversal along with the pressure gradient of the curved pipe and liquid holdup at the outlet are used to determine the onset of liquid-unloading.

Due to the gravity and the curved pipe configuration, the bottom of the curved pipe has a thicker liquid film, which needs a higher gas velocity to unload the liquid film, as shown in Figure 3. When the liquid film at the top of the curved pipe starts to reverse, the bottom liquid film in the pipe is still flowing downward/backward. For the thicker liquid film to be carried to the wellhead a higher gas velocity is required. For this reason, the bottom liquid film reversal is used for the criterion liquid-unloading initiation.

Figure 4 shows the $1 \mathrm{~m}$ curved pipe pressure gradient against the superficial gas velocity with different superficial liquid velocities. The critical gas velocity $V_{\mathrm{sgCR}}$ is demonstrated as the long-dashed line, and the transition of slug flow to annular flow is demonstrated as the short-dashed line. The flow pattern transition has been visually observed and made reference to the flow pattern map by Shoham (2006) and recorded by a high-speed camera.

The pressure gradient consists of gravitational, frictional and accelerated pressure gradients. The accelerated pressure gradient is relatively small compared to the other two terms so that it can be neglected. The superficial gas velocity is low at first, and the total pressure gradient is mainly affected by gravity; as the superficial gas velocity becomes higher, the slug flow shifted to the annular flow pattern, and friction becomes the dominant factor. Like the inclined pipe pressure gradient curve, the critical gas velocity appears slightly earlier than the minimum pressure-gradient point (Liu et al., 2018).

$V_{\mathrm{sg}}$ was relatively low at the beginning of the experiments, the gas phase and liquid phase appeared as the slug flow in the curved pipe and there was liquid loading at the 


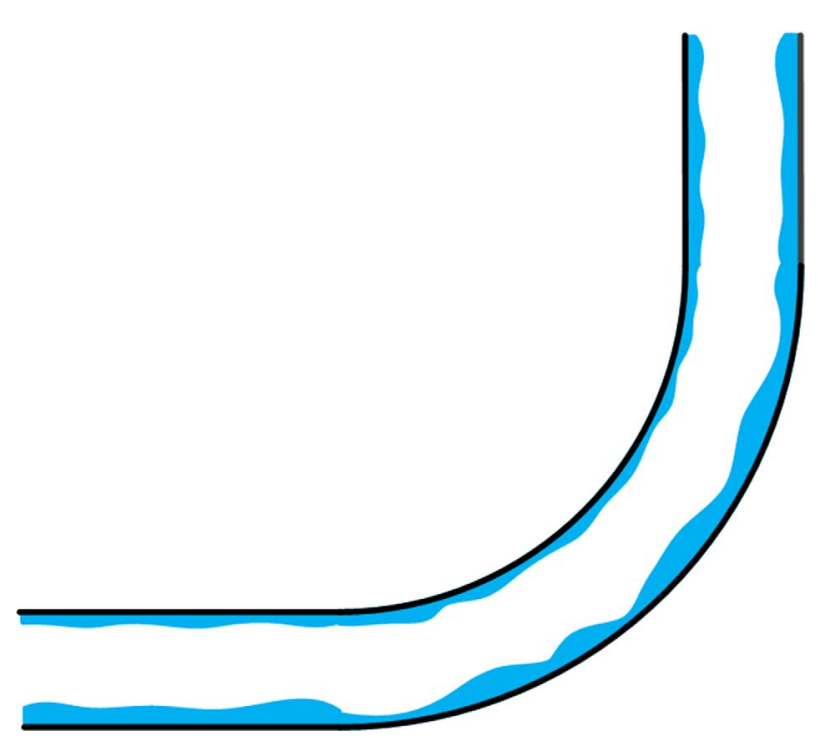

Fig. 3. Schematic illustration of the annular flow in a curved pipe.

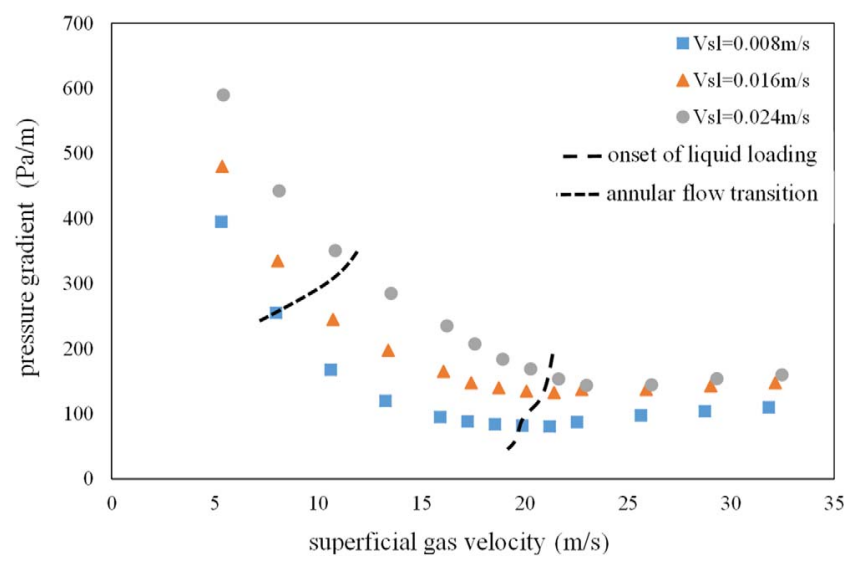

Fig. 4. The pressure gradients against superficial velocities in a $1 \mathrm{~m}$ curved pipe.

bottom of the pipe shown in Figure 5a. As $V_{\text {sg }}$ increased, the gas occupied more space in the curved pipe and formed the gas core, the slug flow shifted to the annular flow shown in Figure 5b. Then, the liquid film became thin and began to move upward carried by the gas core.

It can be found that the transition happens later as the superficial liquid velocity becomes higher. According to the experimental results, for the 3 different superficial liquid velocities $(0.008 \mathrm{~m} / \mathrm{s}, 0.016 \mathrm{~m} / \mathrm{s}$ and $0.024 \mathrm{~m} / \mathrm{s})$, the critical gas velocities $V_{\mathrm{sgCR}}$ are $18.62 \mathrm{~m} / \mathrm{s}, 19.25 \mathrm{~m} / \mathrm{s}$ and $20.20 \mathrm{~m} / \mathrm{s}$ respectively. It can be inferred that the critical gas velocities increase with the superficial liquid velocity.

As the superficial gas velocity rises, the liquid holdup will drop off and display a flat trend (presented in Fig. 6). The right dash line denotes the initiation of liquid film reversal, which is also the criterion of critical gas velocity. For the 3 different superficial liquid velocities $(0.008 \mathrm{~m} / \mathrm{s}$, $0.016 \mathrm{~m} / \mathrm{s}$ and $0.024 \mathrm{~m} / \mathrm{s})$, the critical gas velocity $V_{\mathrm{sgCR}}$ is the same as the results of the pressure gradient.

\subsection{Test results for the curved pipe with $1.5 \mathrm{~m}$ curvature}

Figure 7 shows the pressure gradient against the superficial gas velocity in a $1.5 \mathrm{~m}$ curved pipe. As shown in Figure 7, there is a shift of liquid unloading onset to the right compared to the $1 \mathrm{~m}$ curved pipe case, which means the liquid unloading occurs at a higher critical gas velocity. The flow pattern was also recorded by a high-speed camera. The liquid film is thicker in the $1.5 \mathrm{~m}$ curved pipe than the $1 \mathrm{~m}$ pipe case at the bottom of the curved pipe. Figure 7 shows that for the 3 different superficial velocities $(0.008 \mathrm{~m} / \mathrm{s}, 0.016 \mathrm{~m} / \mathrm{s}$ and $0.024 \mathrm{~m} / \mathrm{s})$, the critical gas velocities $V_{\mathrm{sgCR}}$ are $19.19 \mathrm{~m} / \mathrm{s}, 20.20 \mathrm{~m} / \mathrm{s}$ and $21.50 \mathrm{~m} / \mathrm{s}$ respectively.

Figure 8 shows the liquid holdup against the superficial gas velocity in a $1.5 \mathrm{~m}$ curved pipe. As the superficial gas velocity rises, the liquid holdup shows a similar trend to the $1 \mathrm{~m}$ curved pipe case.

\subsection{Test results for the curved pipe with $2 \mathrm{~m}$ curvature}

Figure 9 shows the pressure gradient against the superficial gas velocity in a $2 \mathrm{~m}$ curved pipe. It shows that the onset of liquid unloading shifts to the right even further with increasing curvature of test pipe, which means the liquidunloading occurs at a higher critical gas velocity than the $1 \mathrm{~m}$ and $1.5 \mathrm{~m}$ curved pipe cases. The flow pattern was also recorded by a high-speed camera. There is a thicker liquid film at the bottom of the $2 \mathrm{~m}$ curved pipe compared to the $1 \mathrm{~m}$ case and $1.5 \mathrm{~m}$ case. Figure 9 shows that for the 3 different superficial velocities $(0.008 \mathrm{~m} / \mathrm{s}, 0.016 \mathrm{~m} / \mathrm{s}$ and $0.024 \mathrm{~m} / \mathrm{s}$ ), the critical gas velocities $V_{\mathrm{sgCR}}$ are $21.10 \mathrm{~m} / \mathrm{s}$, $21.49 \mathrm{~m} / \mathrm{s}$ and $23.00 \mathrm{~m} / \mathrm{s}$ respectively. It can be inferred from the three cases (pipe curvatures are $1 \mathrm{~m}, 1.5 \mathrm{~m}$ and $2 \mathrm{~m}$, respectively) that the critical gas velocity increases with the pipe curvature.

Figure 10 shows the liquid holdup against the superficial gas velocity in a $2 \mathrm{~m}$ curved pipe. It can be found from Figures 9 and 10 that the trend is similar to the $1 \mathrm{~m}$ and $1.5 \mathrm{~m}$ curved pipe cases. The annular flow transitions of the different curved pipes have little difference. With regard to the liquid unloading onsets of different curved pipes, the critical gas velocities increase with the pipe curvatures.

Figure 11 shows the critical gas velocities against the pipe curvatures with different superficial liquid velocities (the measured experimental points show as scattering points). The bar is set to 0.5 . The critical gas velocity increases with the pipe curvature. The critical gas velocity increase trends are linear when the $V_{\mathrm{sl}}=0.016 \mathrm{~m} / \mathrm{s}$ and $V_{\mathrm{sl}}=0.024 \mathrm{~m} / \mathrm{s}$. However, in the $V_{\mathrm{sl}}=0.008 \mathrm{~m} / \mathrm{s}$ case, the critical gas velocity increases slowly at first, then, increases fast as the pipe curvature reaches $2 \mathrm{~m}$.

Figure 12 shows the critical gas velocities against liquid superficial velocities with different pipe curvatures (the measured experimental points show as scattering points). It can be found that the critical gas velocity increases with the liquid superficial velocity rise in all pipe curvature cases. The critical gas velocity increase trends are approximately linear when the pipe curvature is $1 \mathrm{~m}$ and $1.5 \mathrm{~m}$. 
(a)

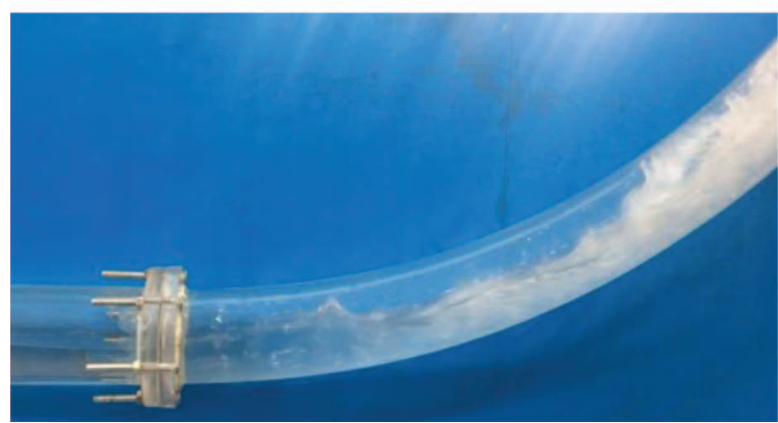

(b)

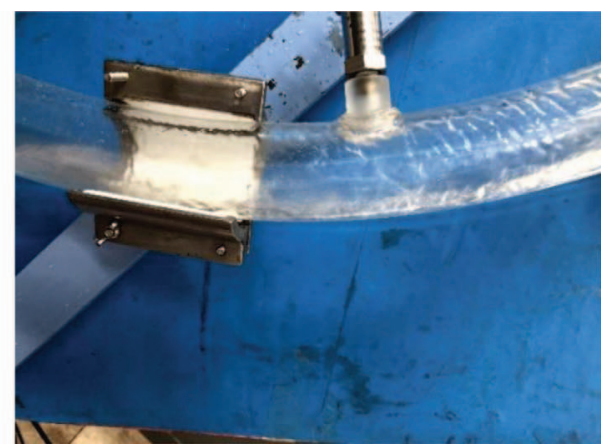

Fig. 5. Different flow patterns in a curved pipe. (a) The slug flow pattern in $1 \mathrm{~m}$ curved pipe. (b) The annular flow in $1 \mathrm{~m}$ curved pipe.

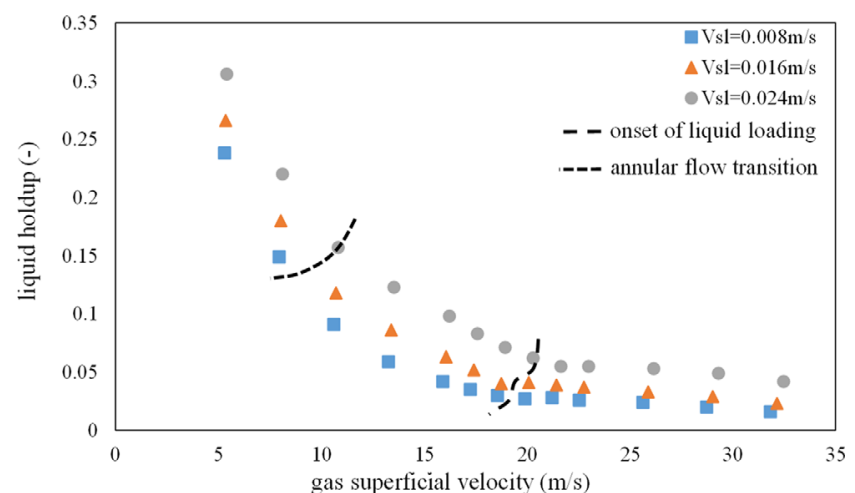

Fig. 6. The liquid holdups against superficial velocities in a $1 \mathrm{~m}$ curved pipe.

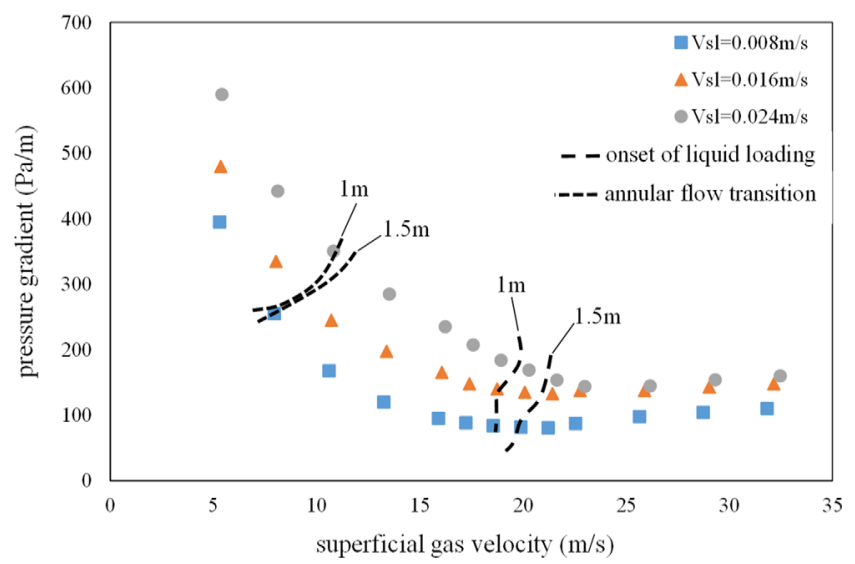

Fig. 7. The pressure gradients against superficial velocities in a $1.5 \mathrm{~m}$ curved pipe.

The critical gas velocity increases slowly at first, and then, increases rapidly when the pipe curvature is $2 \mathrm{~m}$.

\subsection{Analyzing of pipe curvature influence}

The curved pipe can be approximately divided into plenty of straight segments with different inclined angles as shown in Figure 13. Based on a previous study by

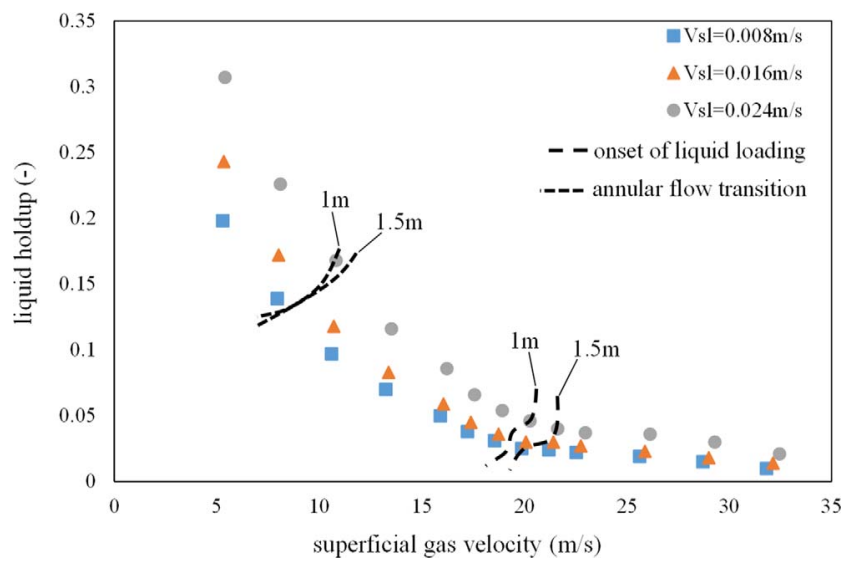

Fig. 8. The liquid holdup against superficial velocities in a 1.5 m curved pipe.

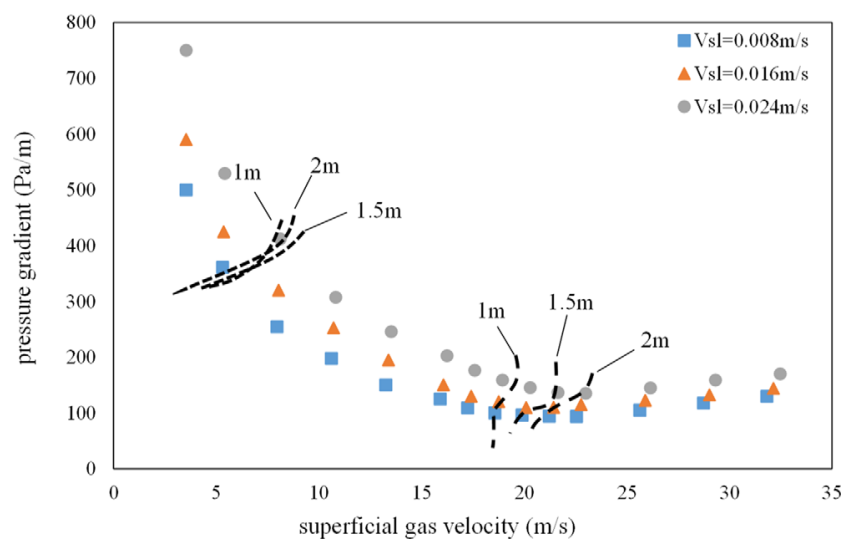

Fig. 9. The pressure gradients against superficial velocities in a $2 \mathrm{~m}$ curved pipe.

Sarica et al. (2013), the $V_{\mathrm{sgCR}}$ increases with the inclined angle (reach the maximum critical gas velocity at the inclined angle of $30^{\circ}$ ) and decreases with the increase of the inclined angle. It is mainly due to the balance between the gas drag force and gravitational force. $\theta$ is the angle between the inclined segment and the horizontal in equation (1). At the small angle, the increase rate of $\sin \theta$ is larger than the decrease rate of $g_{f}$ while the angle 


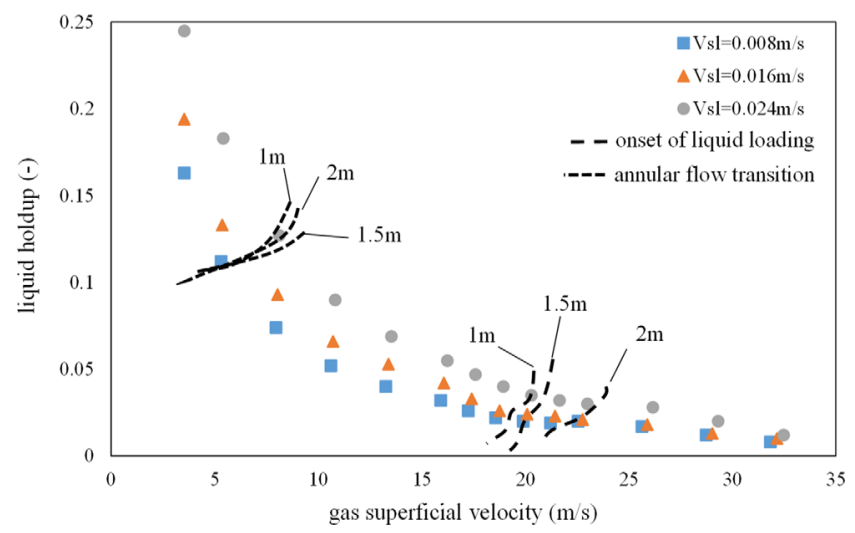

Fig. 10. The liquid holdup against superficial velocities in a $2 \mathrm{~m}$ curved pipe.

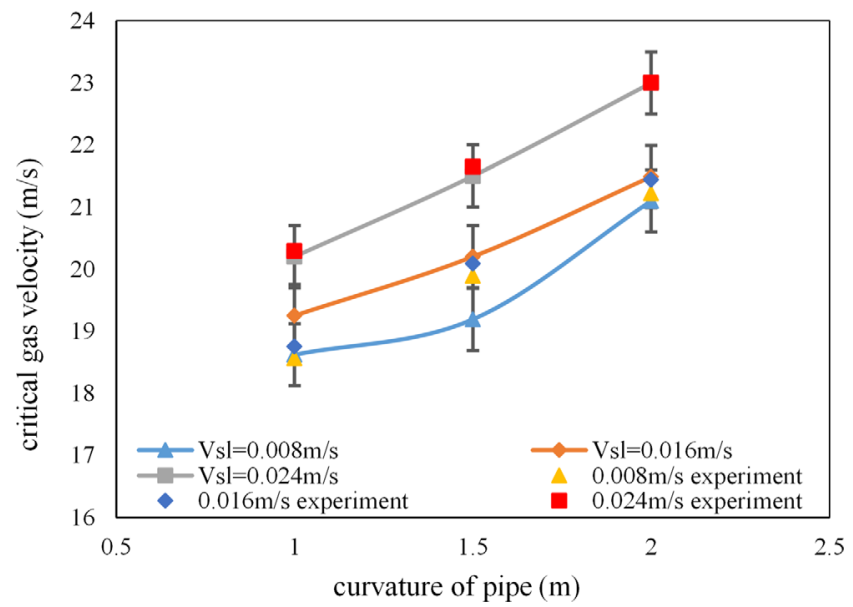

Fig. 11. The curvature of pipes against critical gas velocities.

increases, which means there is a larger $F_{g}$ for preventing the liquid film from falling. However, as the inclined angle reaches a certain value, the angle's increase will result in a smaller $F_{g}$ :

$$
F_{g}=g_{f} \sin \theta .
$$

As can be seen from the analysis of the approximate curved pipe that consists of several inclined segments, the liquid unloading mechanism will be much more complicated in an actual curved pipe. There will be different critical gas velocities at different locations, and the centrifugal force will lead to the variation of liquid film thickness at the bottom of the pipe. As illustrated in equation (2), the centrifugal force is related to the liquid velocities and the pipe curvature:

$$
F_{c}=m_{f} \frac{V_{f}^{2}}{r} .
$$

For a constant pipe curvature, the centrifugal force increases with the liquid film velocity; and for a constant liquid film velocity, the centrifugal force decreases as the pipe curvature becomes larger. The liquid film will become thicker when the centrifugal force decreases.

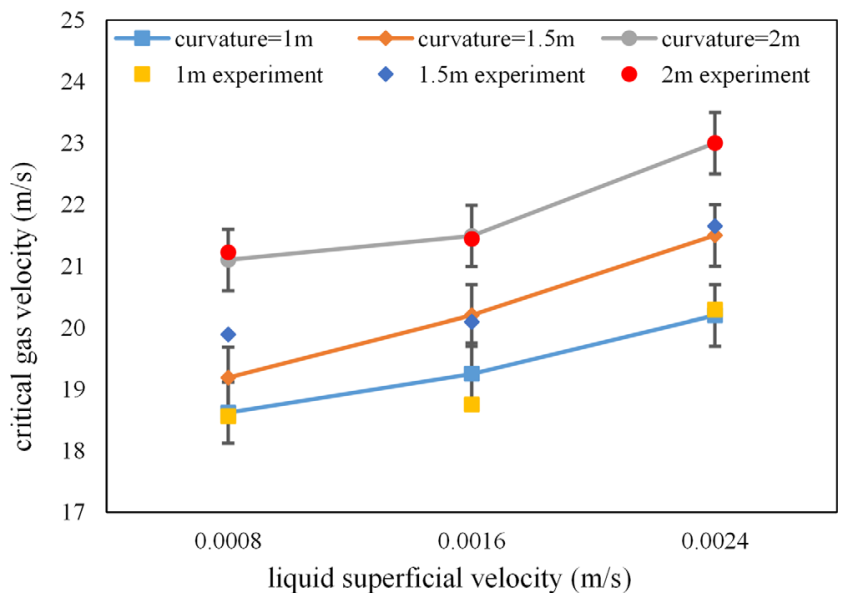

Fig. 12. The liquid superficial velocities against critical gas velocities.

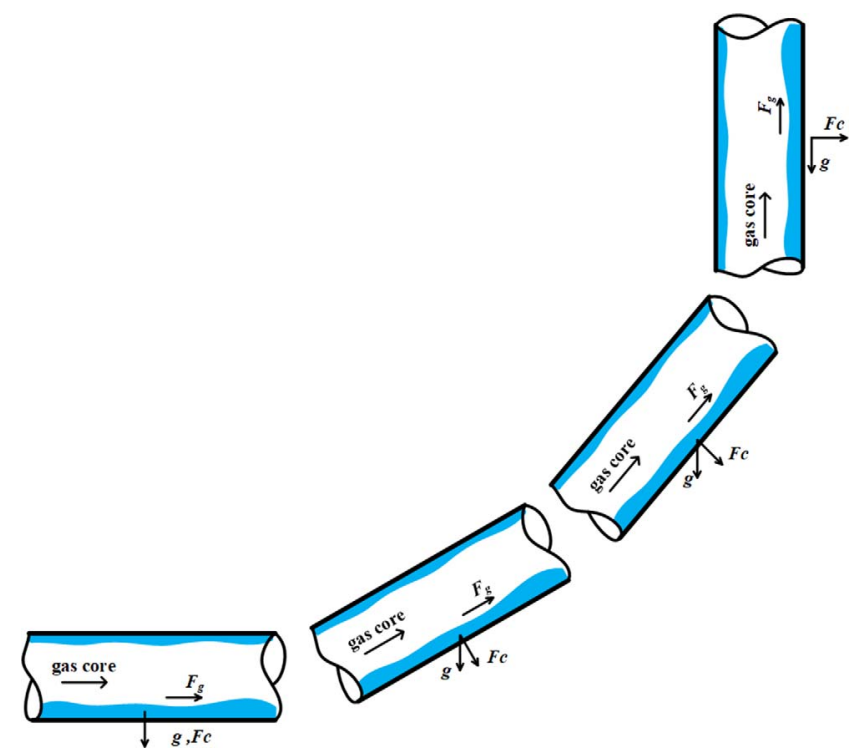

Fig. 13. The flow direction and force balance of the liquid film in divided segments of a curved pipe.

\section{Model comparison}

The Beggs-Brill method (Beggs and Brill, 1973) and the OLGA model (Bendiksen et al., 1991) are used to calculate the pressure gradient and liquid holdup in curved pipes, experimental data are compared to the models' calculations. The comparison purpose is to find out whether these two models can accurately predict the liquid unloading process in a curve pipe. The OLGA model and Beggs-Brill model are applied only to the straight pipe. Therefore, in the curve pipe case, the curve pipe is divided into several inclined pipes, then, the pressure and liquid holdup segment by segment from outlet to inlet are calculated (shown as Fig. 13). The used experimental pressure gradient data are from the inlet and the outlet of the curve pipe. The used experimental liquid holdup data are from the outlet of the 


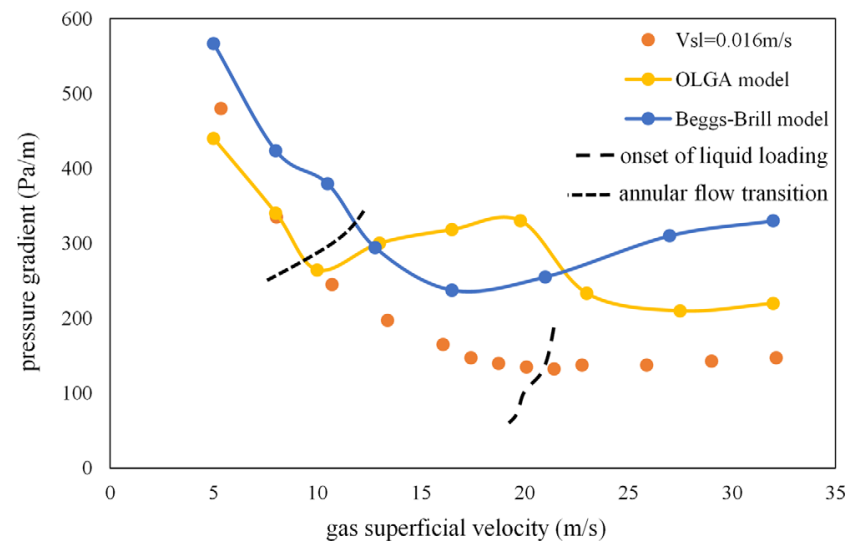

Fig. 14. The pressure gradient comparisons between models and experimental data in the $1.5 \mathrm{~m}$ curved pipe with superficial liquid velocity $0.016 \mathrm{~m} / \mathrm{s}$.

curve pipe. Then, the error analysis is done to evaluate whether these two models fit the liquid unloading process in a curved pipe.

\subsection{The pressure gradient comparison}

Pressure gradient data are compared with the calculated results of the Beggs-Brill model and the OLGA model. The experimental data of $1.5 \mathrm{~m}$ and $2 \mathrm{~m}$ pipe curvature cases are chosen for the comparisons.

Figure 14 presents the predicted pressure gradients and experimental data in the $1.5 \mathrm{~m}$ curved pipe with the superficial liquid velocity $V_{\mathrm{sl}}=0.016 \mathrm{~m} / \mathrm{s}$. At the low gas superficial velocity, the prediction of the OLGA model is rather well, and the result of the Beggs-Brill model is much larger than the experimental data. As the gas superficial velocity increases, the flow pattern changes into the annular flow, and it is found that the errors between the two models and experimental data are very large.

Figure 15 shows the predicted pressure gradients and experimental data in the $2 \mathrm{~m}$ curved pipe with the superficial liquid velocity $V_{\mathrm{sl}}=0.016 \mathrm{~m} / \mathrm{s}$. It is similar to the $1.5 \mathrm{~m}$ case, at the low gas superficial velocity, the predictions of the OLGA model and Beggs-Brill model are fitted to experimental data relatively well. As the gas superficial velocity rises, the predicted results of both models are larger than the experimental data. It is found that the errors between the calculated results and experimental data also are very large.

Figure 16 presents the predicted pressure gradients and experimental data in the $1.5 \mathrm{~m}$ curved pipe with the superficial liquid velocity $V_{\mathrm{sl}}=0.024 \mathrm{~m} / \mathrm{s}$. Similar to the $V_{\mathrm{sl}}=0.016 \mathrm{~m} / \mathrm{s}$ case, at the low gas superficial velocity, the prediction of the OLGA model is better than the Beggs-Brill model's prediction. As the gas superficial velocity increases, the deviations between the two models and experimental data are very obvious.

Figure 17 presents the predicted pressure gradients and experimental data in the $2 \mathrm{~m}$ curved pipe with the superficial liquid velocity $V_{\mathrm{sl}}=0.024 \mathrm{~m} / \mathrm{s}$. At the low gas

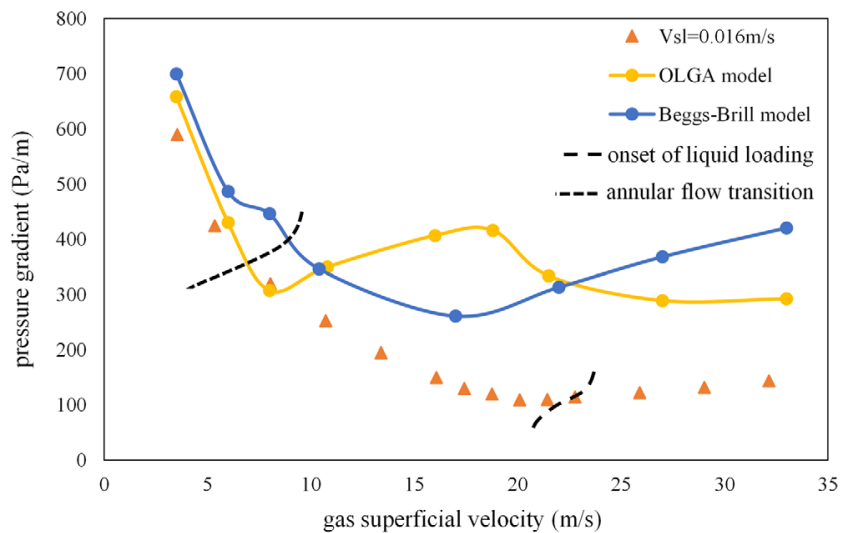

Fig. 15. The pressure gradient comparisons between models and experimental data in the $2 \mathrm{~m}$ curved pipe with superficial liquid velocity $0.016 \mathrm{~m} / \mathrm{s}$.

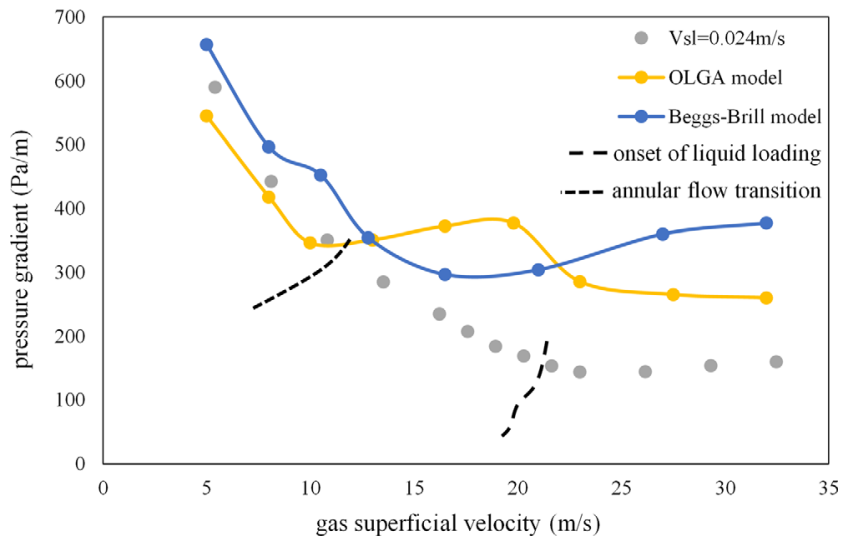

Fig. 16. The pressure gradient comparisons between models and experimental data in the $1.5 \mathrm{~m}$ curved pipe with superficial liquid velocity $0.024 \mathrm{~m} / \mathrm{s}$.

superficial velocity, the prediction of the OLGA model fits rather well to the experimental data. However, at the high gas superficial velocity, the prediction errors between the two models and experimental data are very large.

The comparisons of experimental pressure gradient data and the predictions of the OLGA model and Beggs-Brill model are shown in Figure 18. The left dash line is the $-50 \%$ error bar of experimental data. The right dash line is the $+50 \%$ error bar of experimental data. According to the error analysis result, most of the prediction results are larger than the experimental data, and the model calculation accuracy of the $1.5 \mathrm{~m}$ case is slightly better than the model calculation accuracy of the $2 \mathrm{~m}$ case. However, in general, both the OLGA model and Beggs-Brill model do not show good agreement with the experiments.

\subsection{The liquid holdup comparison}

Predicted liquid holdups and experimental data in the $1.5 \mathrm{~m}$ curved pipe with the superficial liquid velocity $V_{\mathrm{sl}}=0.016 \mathrm{~m} / \mathrm{s}$ are shown in Figure 19. At the low gas 


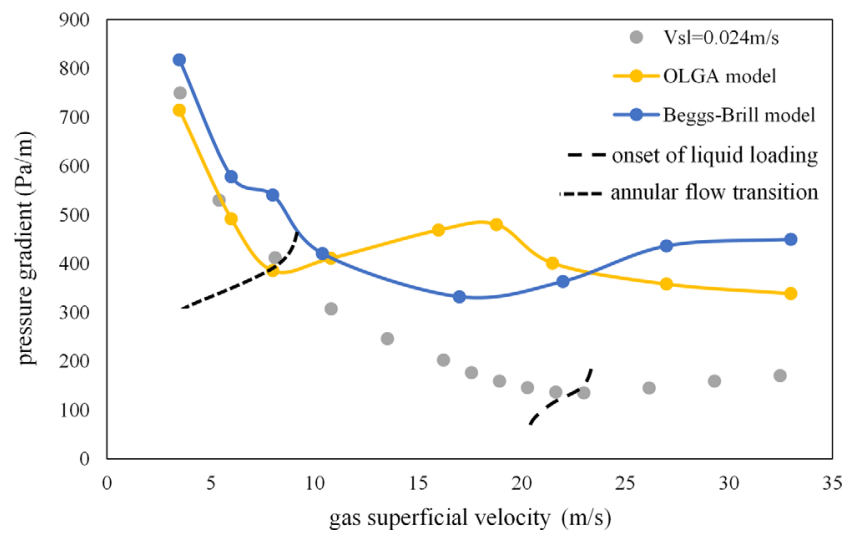

Fig. 17. The pressure gradient comparisons between models and experimental data in the $2 \mathrm{~m}$ curved pipe with superficial liquid velocity $0.024 \mathrm{~m} / \mathrm{s}$.

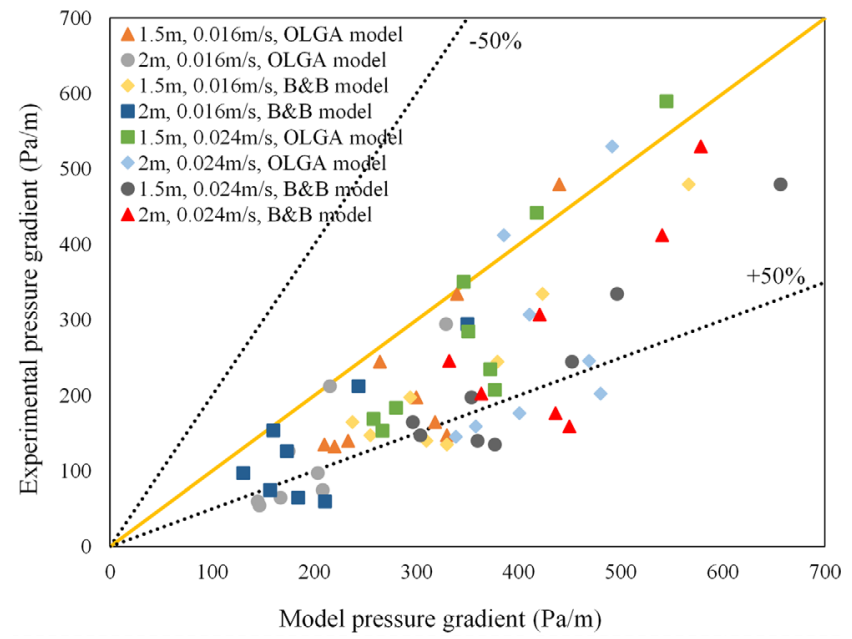

Fig. 18. The overall comparisons between models and experimental data.

superficial velocity, the OLGA model and the Beggs-Brill model predicted well compared to experimental data. As the superficial gas velocity rises, the flow pattern changes and it shows a turning point in the predicted curves. It can be found that the differences between the two models and experimental data become large, the errors between the predictions of the Beggs-Brill model and experimental data are bigger than the case of the OLGA model.

Predicted liquid holdup curves and experimental data in the $2 \mathrm{~m}$ curved pipe with the superficial liquid velocity $V_{\mathrm{sl}}=0.016 \mathrm{~m} / \mathrm{s}$ are shown in Figure 20. At the low gas superficial velocity, the OLGA model and the Beggs-Brill model predicted results are obviously larger than the experimental data; as the gas superficial velocity rises, the curves are similar to the $1.5 \mathrm{~m}$ case, there is a turning point at the flow pattern transition of the predicted curves. It can be found that the differences between the Beggs-Brill model and experimental data become larger, and the OLGA

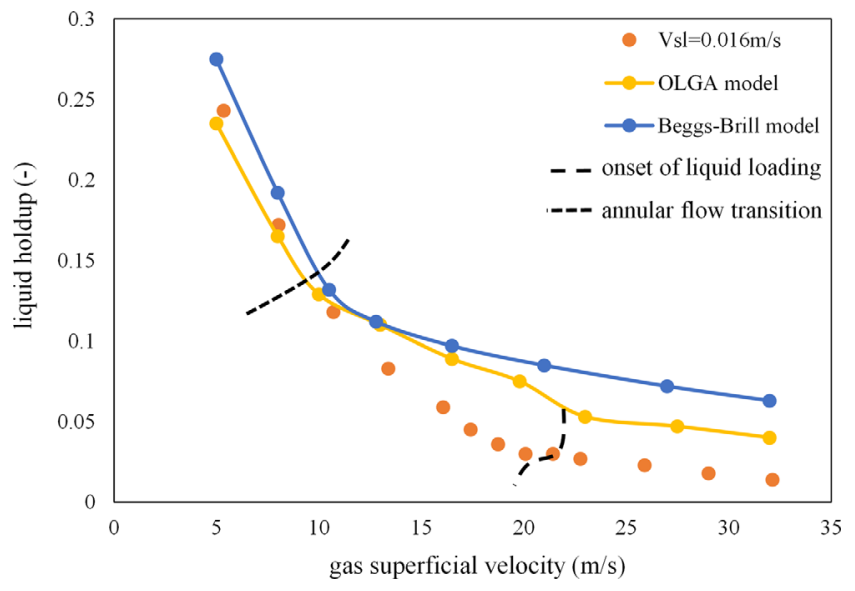

Fig. 19. The liquid holdup comparisons between models and experimental data in the $1.5 \mathrm{~m}$ curved pipe with superficial liquid velocity $0.016 \mathrm{~m} / \mathrm{s}$.

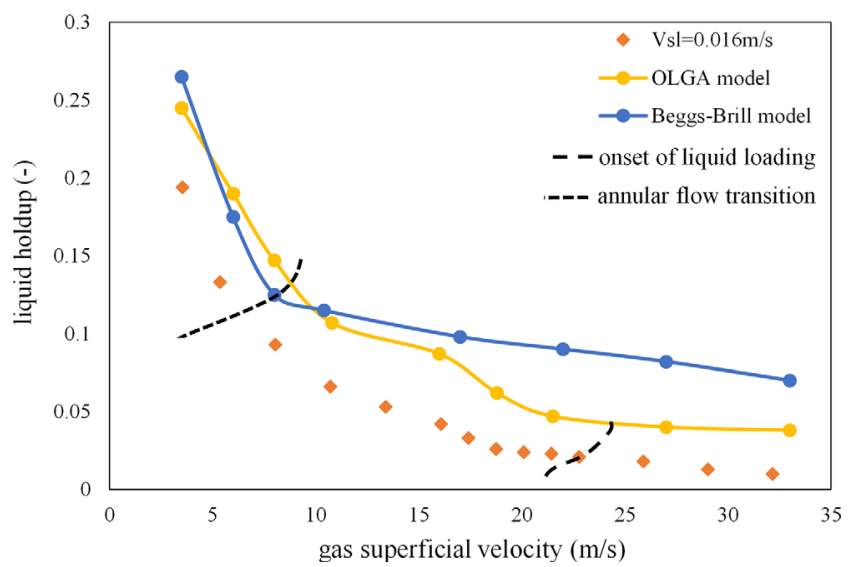

Fig. 20. The liquid holdup comparisons between models and experimental data in the $2 \mathrm{~m}$ curved pipe with superficial liquid velocity $0.016 \mathrm{~m} / \mathrm{s}$.

model prediction is fitted relatively better with the experimental data than the Beggs-Brill model.

The comparisons of experimental liquid holdup data and the predictions of the OLGA model and Beggs-Brill model in the $1.5 \mathrm{~m}$ curve pipe with the superficial liquid velocity $V_{\mathrm{sl}}=0.024 \mathrm{~m} / \mathrm{s}$ are shown in Figure 21. It can be found that the prediction deviation of the Beggs-Brill model is larger than the prediction of the OLGA model of the whole range.

The comparisons of experimental liquid holdup data and the predictions of the OLGA model and Beggs-Brill model in the $2 \mathrm{~m}$ curve pipe with the superficial liquid velocity $V_{\mathrm{sl}}=0.024 \mathrm{~m} / \mathrm{s}$ are shown in Figure 22 . It can be found that the errors between the prediction of the two models and the experimental data are large of the whole range.

Figure 23 presents the comparisons of experimental liquid holdup data and the predictions of the OLGA model and Beggs-Brill model. The left dash line is the $-50 \%$ error bar of experimental data. The right dash line is the 


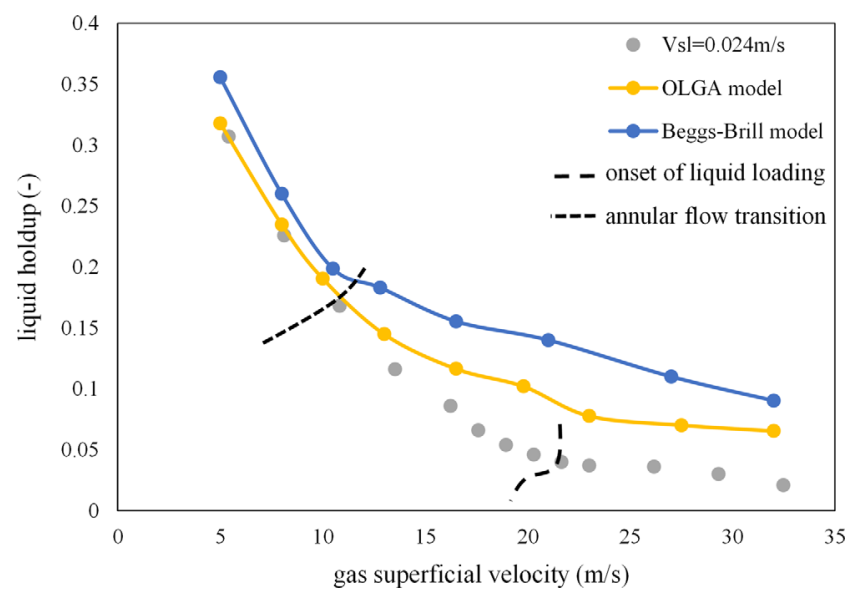

Fig. 21. The liquid holdup comparisons between models and experimental data in the $1.5 \mathrm{~m}$ curved pipe with superficial liquid velocity $0.024 \mathrm{~m} / \mathrm{s}$.

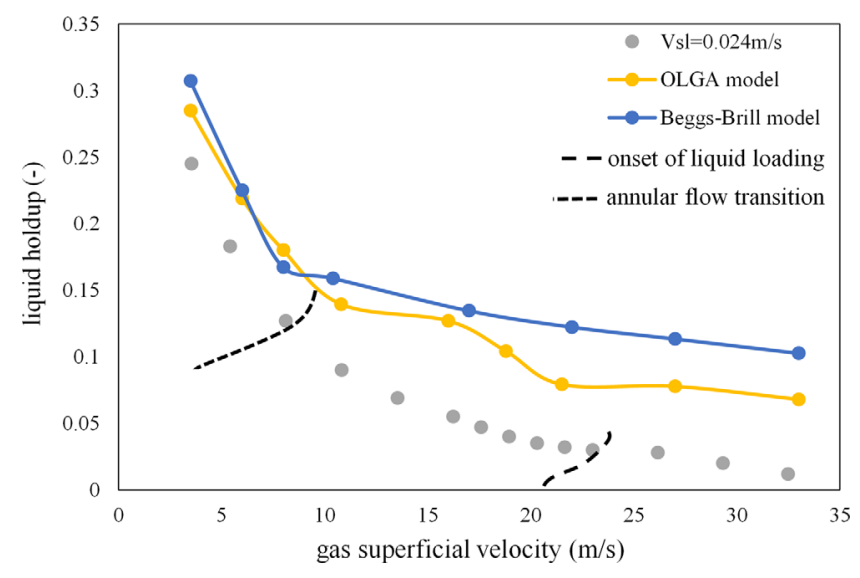

Fig. 22. The liquid holdup comparisons between models and experimental data in the $2 \mathrm{~m}$ curved pipe with superficial liquid velocity $0.024 \mathrm{~m} / \mathrm{s}$.

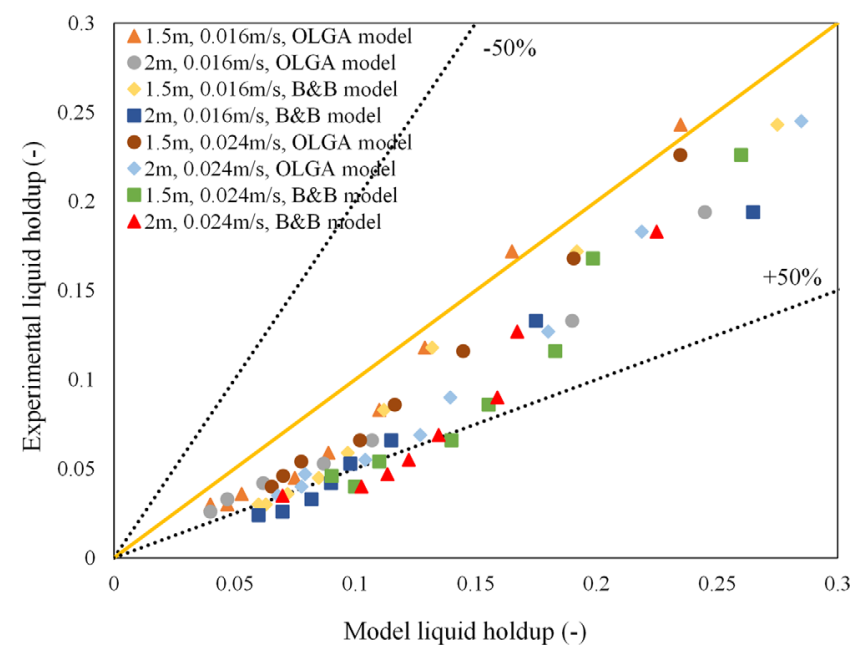

Fig. 23. The liquid holdup overall comparisons between models and experimental data.
$+50 \%$ error bar of experimental data. According to the error analysis result, most of the prediction results are larger than the experimental data and in the range of $0-50 \%$. The liquid holdup result is similar to the pressure gradient situation, the accuracy of the $1.5 \mathrm{~m}$ case is slightly better than the accuracy of the $2 \mathrm{~m}$ case, and both the OLGA model and Beggs-Brill model do not show good agreement with the experiments.

One of the main reasons for the discrepant results of the models is that the OLGA model and Beggs-Brill model are applied only to the straight pipe. Moreover, it is difficult to predict liquid loading initiation in a curved pipe under various situations. Our next step is building the theoretical model combined with this experimental result to give guidance to gas production.

\section{Conclusion}

In this study, experiments of the liquid unloading process in the curved pipe with different pipe curvatures and superficial liquid and gas velocities were conducted. The pressure and liquid holdup of the curved pipe were measured to analyze the critical gas velocity. Two models' predictions were compared with the experimental data to verify the accuracy. The following conclusions can be drawn:

1. The different curved pipe experimental results indicate that the critical gas velocity increases with the increase of pipe curvature. The increasing critical gas velocity trend is mainly due to the smaller centrifugal force in the larger curvature pipe, which will lead to the increase of liquid film thickness, and it means the liquid carrying capacity is reducing.

2. From the different liquid velocity experimental data, the critical gas velocity increases with the superficial liquid velocity rise in all the pipe curvature cases (pipe curvature radius is $1 \mathrm{~m}, 1.5 \mathrm{~m}$ and $2 \mathrm{~m}$, respectively).

3. The pressure gradient and liquid holdup of $1.5 \mathrm{~m}$ case and $2 \mathrm{~m}$ case were selected to compare with the prediction results of the OLGA model and Beggs-Brill model. Both the models fit relatively well to the experimental data at the low superficial gas velocity. As the superficial gas velocity rises, the OLGA model fits better to the experimental data than the Beggs-Brill model. The $1.5 \mathrm{~m}$ cases of both models fit better than the $2 \mathrm{~m}$ cases.

4. The error analysis shows that overall the predicted results are not in good agreement with experimental data. Several errors between experimental data and predictions are over $50 \%$.

5. According to the comparison results between current models and experimental data, further efforts on the theoretical model of liquid unloading in the curved pipe are strongly needed.

Acknowledgments. The authors express gratitude for the CNPC project "Research on key technologies for economic and effective development of extra-low permeability carbonate reservoir" (Grant number: 2021DJ3202). 


\section{References}

Anderson R.J., Russell T.W.F. (1970) Film formation in twophase annular flow, AIChE J. 16, 626-633.

Azzopardi B.J., Piearcey A., Jepson D.M. (1991) Drop size measurements for annular two-phase flow in a $20 \mathrm{~mm}$ diameter vertical tube, Exp. Fluids 11-11, 191-197.

Beggs D.H., Brill J.P. (1973) A study of two-phase flow in inclined pipes, J. Petrol. Technol. 25, 607-617.

Belfroid S., Schiferli W., Alberts G., Veeken C.A.M., Biezen E. (2008) Predicting onset and dynamic behaviour of liquid loading gas wells, in: SPE Annual Technical Conference and Exhibition, Society of Petroleum Engineers.

Bendiksen K.H., Maines D., Moe R., Nuland S. (1991) The dynamic two-fluid model OLGA: Theory and application, SPE Prod. Eng. 6, 171-180.

Coleman S.B., Clay H.B., McCurdy D.G., Norris L.H. (1991) Understanding gas-well load-up behavior, J. Petrol. Technol. 43, 334-338.

Fossa M. (1998) Design and performance of a conductance probe for measuring the liquid fraction in two-phase gas-liquid flows, Flow Meas. Instrum. 9, 103-109.

Greene W.R. (1983) Analyzing the performance of gas wells, J. Petrol. Technol. 35, 1378-1384.

Guner M., Pereyra E., Sarica C., Torres C. (2015) An experimental study of low liquid loading in inclined pipes from 90 to 45, in: SPE Production and Operations Symposium, Society of Petroleum Engineers.

Klumpner C., Blaabjerg F. (2002) Experimental evaluation of ride-through capabilities for a matrix converter under short power interruptions, IEEE Trans. Ind. Electron. 49, 315-324.

Landjobo Pagou A., Han G., Peng L., Dehdah O., Gueyap Kamdem V., Abimbola F., Mccarthy S.A., Tchomche H.F., Harmash I., Kanturina Z. (2020) Liquid loading prediction and identification model for vertical and inclined gas wells, J. Nat. Gas Sci. Eng. 84, 103641.

Lea J.F., Nickens H.V. (2004) Solving gas-well liquid-loading problems, J. Petrol. Technol. 56, 30-36.

Lea J.F., Nickens H.V., Wells M.R. (2008) Gas well deliquification, Gulf Professional Publishing.

Lea J.F., Rowlan L. (2019) Nodal analysis, in: Gas well deliquification, pp. 37-45.

Liu Y., Luo C., Zhang L., Liu Z., Xie C., Wu P. (2018) Experimental and modeling studies on the prediction of liquid loading onset in gas wells, J. Nat. Gas Sci. Eng. 57, 349-358.

Min L., Lei S., Shilun L. (2001) New view on continuous-removal liquids from gas wells, in: Proceedings of SPE Permian Basin Oil and Gas Recovery Conference, Society of Petroleum Engineers.

Nosseir M.A., Darwich T.A., Sayyouh M.H., El Sallaly M. (2000) A new approach for accurate prediction of loading in gas wells under different flowing conditions, SPE Prod. Facil. 15, 241-246.

Pagan E.V., Waltrich P.J. (2016) A simplified model to predict transient liquid loading in gas wells, J. Nat. Gas Sci. Eng. 35, $372-381$.
Riza M.F., Hasan A.R., Kabir C.S. (2016) A pragmatic approach to understanding liquid loading in gas wells, SPE Prod. Operat. 31, 185-196.

Rodrigues H.T., Pereyra E., Sarica C. (2018) A model for the thin film friction factor in near-horizontal stratified-annular transition two-phase low liquid loading flow, Int. J. Multiph. Flow 102, 29-37.

Sarica C., Yuan G., Sutton R.P., Pereyra E.J. (2013) An experimental study on liquid loading of vertical and deviated gas wells, in: SPE Production and Operations Symposium, Society of Petroleum Engineers, pp. 158-161.

Sawai T., Kaji M., Kasugai T., Nakashima H., Mori T. (2004) Gas-liquid interfacial structure and pressure drop characteristics of churn flow, Exp. Thermal. Fluid Sci. 28, 597-606.

Shoham O. (2006) Mechanistic modeling of gas-liquid two-phase flow in pipes, Society of Petroleum Engineers, USA.

Shekhar S., Kelkar M., Hearn W.J., Hain L.L. (2017) Improved prediction of liquid loading in gas wells, SPE Prod. Operat. 32, 539-550.

Shi S., Wu X., Han G., Zhong Z., Li Z., Sun K. (2019) Numerical slug flow model of curved pipes with experimental validation, ACS Omega 4, 12, 14831-14840.

Skopich A., Pereyra E., Sarica C., Kelkar M. (2015) Pipediameter effect on liquid loading in vertical gas wells, SPE Prod. Operat. 30, 164-176.

Turner R.G., Hubbard M.G., Dukler A.E. (1969) Analysis and Prediction of Minimum Flow Rate for the Continuous Removal of Liquids from Gas Wells, J. Petrol. Technol. 21, 1475-1482.

van't Westende J.M.C., Kemp H.K., Belt R.J., Portela L.M., Mudde R.F., Oliemans R.V.A. (2007) On the role of droplets in cocurrent annular and churn-annular pipe flow, Int. J. Multiph. Flow 33, 595-615.

Waltrich P.J., Posada C., Martinez J., Falcone G., Barbosa J.R. (2015) Experimental investigation on the prediction of liquid loading initiation in gas wells using a long vertical tube, J. Nat. Gas Sci. Eng. 26, 1515-1529.

Wang Z., Bai H., Zhu S., Zhong H., Li Y. (2015) An entraineddroplet model for prediction of minimum flow rate for the continuous removal of liquids from gas wells, SPE J. 20, 1135-1144.

Williams L.R., Dykhno L.A., Hanratty T.J. (1996) Droplet flux distributions and entrainment in horizontal gas-liquid flows, Int. J. Multiph. Flow 22, 1-18.

Zhong Z., Wu X., Han G., Li C., He L., Xiong X., Wang S. (2018) Experimental investigation on particle transport of coal fines in unsteady terrain slug flow, J. Pet. Sci. Eng. 166, 747-758.

Zhou C., Wu X., Zhang T., Zhao X., Gai S., Xiang H. (2019) Dynamic analysis for two-phase vortex flow and optimization of vortex tools to unload liquid from gas wells, J. Pet. Sci. Eng. 173, 965-974.

Zhou C., Wu X., Li H., Lin H., Liu X., Cao M. (2016) Optimization of methods for liquid loading prediction in deep condensate gas wells, J. Pet. Sci. Eng. 146, 71-80. 


\section{Appendix}

\section{The conductivity device calibration}

A measurement unit consists of 4 stainless steel conductivity rings which are $10 \mathrm{~mm}$ wide. The conductivity ring has the same diameter as the pipes, which will have no influence on the flow. Every two conductivity rings consist of a measure group (G1 and G2). The more liquid is in the cross-section, the lower resistance the flow has, which means the voltage is higher, and the larger liquid holdup becomes. The voltage signals are collected by a high-speed acquisition card, then, transformed into numerical signals, and are displayed and stored in the computer.

The calibration procedure is:

1. Wipe off the water at the inner wall of the measurement unit, and keep it dry. Record the voltage signals of $\mathrm{G} 1_{\min }$ and $\mathrm{G} 2_{\min }$, respectively.

2. Filling the measurement unit with water, record the maximum water volume and the voltage $\mathrm{G} 1_{\max }$ and $\mathrm{G} 2_{\max }$.
3. Discharge the water and make the measurement dry.

4. Divide the water equally into 20 parts. Fill the water into the measurement unit part by part, and record the voltage signals $\mathrm{G} 1_{s}$ and $\mathrm{G} 22_{s}$, respectively $(s=1$, $2, \ldots, 19)$.

5. Repeat step (1) to step (4), average the recorded results.

6. Normalize the recorded voltages with the equation (A.1),

$\left(\mathrm{NV}_{\mathrm{Gk}}\right)_{s}=\left\{\begin{array}{l}0, s=0 \\ \frac{\left(V_{\mathrm{Gk}}\right)_{s}-\left(V_{\mathrm{Gk}}\right)_{\min }}{\left(V_{\mathrm{Gk}}\right)_{\max }-\left(V_{\mathrm{Gk}}\right)_{\min }}, s=1,2, L, \quad 19 ; k=1,2 . \\ 1, s=20\end{array}\right.$ 